\title{
Dieter Deiseroth Stationierung amerikanischer Atomwaffen - begrenzte Souveränität der Bundesrepublik? :*
}

\section{A. Problemstellung}

Nach dem Naro-Rats-Beschluß vom 12. Dez. 1979' ist die Stationierung von 108 ballistischen Flugkörpern Pershing II mit einer Reichweite bis zu $2800 \mathrm{~km}$ als Ersatz für die 108 Pershing I der US-Armee in der Bundesrepublik Deurschland und von 464 aerodynamischen Düsenantriebs-Flugkörpern ("cruise missilesw) für Start vom Boden (GLCM) zu je vier auf einem Startahrzeug ( i 6 fahrbare vierfach Startrampen), insgesami also von 572 Flugkörpern mit je einem nuklearen Gefechrskopf, ab Herbst 1983 geplant. ${ }^{2}$ Diese neuen nuklearen Mittelstreckensyszeme sollen in Europa ausscbließlich in den US-Sireilkräften eingeführt werden.

Die Alternative zu diesem whost-country-model $x$, das user-councry-modelu, war zuerst von der Bundesregierung der Bundesrepublik Deutschland, dann auch von den anderen Dislozierungsländern abgelehnt worden; das abgelehnte Konzept härte bedeuteh »daß die Startgeräte, die Intrastrukruraniagen, die Instandhalrungs- und Betriebskosten für das ganze System vom Dislozierungsland aufgebrachr werden müßten; die Gefechrsköpfe mit den Flugkörpern blieben US-Eigentum und unter US-Verwahrung; eine Zwei-Schlüssel-Xontroilgewalt würde bilateral organisierr; die Bedienungsmannschaften würden unter Aufsicht von amerikanischen »custodians* vom Dislozierungsland gestellt wie bei den üblichen TNF-Systemen *'.

Nach dem nunmehr vorgesehenen shost-country-model $\alpha$ sollen die neuen (amerikanischen) Waffen wie alle anderen nukiearen Waffensysteme der Alliierten Streirkräfte in Europa dem Obersten Allïerten Befehlshaber (SACEUR) unterstellt werden.

Dieser Beschluß der Nato hat in den vergangenen Jahren bekanntermaßen zu einer sehr kontroversen öffentlichen Diskussion und heftigen politischen Auseinanderset-

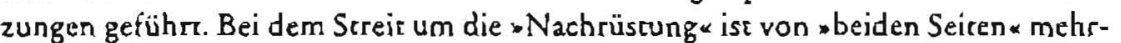
fach die Frage aufgeworfen worden, ob die US-Administration bei einer Ausbreiung der wholländischen Krankheic* unter Umständen auch gegen den Willen der zuständigen Organe der Bundesrepublik die neuen Waffen in der Bundesrepublik stationieren könnte. Sebr oft ist dabei der Begriff von der nach wie vor mbegrenzten Souveränität火 der Bundesrepublik in die Diskussion gebracht woruen.

* Bei dem vorliegenden Beitrag handelt es sich um die gekürzte und leicht veränderte Fassung einec Studie. die der Vertasser unter dem Titel -US-amerikanische Stavionierungstechte und stomare Enescheidungsbefugnisse in des Bundescepublik Deutschland. im Auftrag des -Forschungsinstieuss iür Friedenspolicik c. V. in Suanberg. ersecllt hat; die Studie ist in der Schriftenceihe des Eorschungsinseieues verölfentliche worden (Starmberg Seprember/November 1982).

1 Vel. Jen Texi des Brüsseler Kommuniques der Außen- und Vencidigungsminister vom 12. 12. 79, in; EA ע/1980, S. D is fí.

2 Zur Vorgeschichee Jes Brusseler Beschlusses siche Mikon Leienberg. Die takrischen Nukleanwaffen groBer Reichweite der Nato und der WVO, in: Sudiengruppe Militärpolicik, Aufrüsıen um abzuristen?. Reinbek 1980. S. 28 1f: Lothar Ruchl. Der Beschiuß der Naco zur Einlührung nuklearer Mlittelsececkenwaitun, EA 1980, S. 99 is.

, Vpl. dazu Ruehl, 1. 2. O., S. 109 f. 
Bcsonderen Auftrieb erhielt diese Diskussion durch mebrere Äußenungen des amerikanischen Verteidigungsministers Weinberger, der verlautbart hatte, die Amerikaner hätten das Recht, ihre Truppen *mit dern Besten auszurüsten, was sie hätten *, und niemand könne ihnen das verbieren." Vor allem im Zusammenhang mit der Bckanntgabe der Entscheidung der Reagan-Administration aus dem Jahrc 1981, eine größere Anzahl Neutronensprengköpfe zu produzieren, hazte Weinberger erklärt: - Konsulzarionen über diese Waffe sind cbensowenig erforderlich wie über die Produktion einer konventionellen is5-mm-Artillerie-Granaten; diese Sprengköpfe könnten, natürlich nach Konsultation (nicht: nach Zustimmung) der Empfängerländer, ninnerhalb weniger Stunden « nach Europa geflogen werden und erhöhten dort »dic Verteidigungsstärke der Vereinigten Staten und ihrer europäischen Verbündeten s. $^{3}$

Der Chef-Präsidentenberater Ed. Meese soll nach Presseberichten der deutschen Regierungsdelegation am Rande des Ortawa-Gipfels im Jahre 198 r deutlich gemacht haben, daß w weder die Nato-Vereinbarung noch der Truppenvertrag (vorsehen), da $B$ Washington seine Partner um Erlaubnis fragen muß, wenn bei den US-Streitkräfren in Europa neue Waffen eingeführ werden.* "Nach den geheimen Zusätzen des Truppenscarutsa werde die Bundesregierung vor der Scationierung neuer Aromwaffen zwar konsultiert, aber nach dem Abkommen, so Meese, *können wir machen, was wir wollenk. ${ }^{\circ}$

Von bundesrepublikanischen Politikern waren diese Äußcrungen seinerzeit mir deurlichem Protest aufgenommen worden; so hatre etwa der Frakrionsvorsitzende der EDP, Wolfgang Mischnick, die Amerikaner belehrt, sie müßren s sich im Klaren darüber sein, daß die Zustimmung zur Lagerung (der Neutronenwaffen) eine Entscheidung der europäischen Bündnispartner ist .' $^{\prime}$

In der Zwischenzeit sollen hohe Vertreter der Reagan-Administration, darunter Verteidigungsminister Weinberger, ihren Standpunkt wiche nur niche wiederholt, sondern korrigiert haben . $^{8}$

Allerdings ist die Diskussion über die »begrenzte Souveränität* der BRD seirdem nicht mehr verstummt. Zudem gibr es Berichte, denen zufolge der Bundesregierung in Bonn zwei Gutachten vorliegen sollen (eines im Außenministerium, eines im Verteidigungsministerium erscellt $\rangle$, die zur Frage der Stationierungsbefugnisse der USA zu unterschiedlichen Engebnissen kommen: Das Gutachren aus dem Auswärrigen Amt soll die Frage, ob aufgrund der alten Besatzungsrechre die USA auch ohne Zustimmung der Bundesregierung neue Waffen stationieren können, bejahen; die Expertise aus dem Bundesministerium der Verteidigung soll die Frage verneinen. Ähnlich brisant ist die Frage, ob und $g g f$. in welcher Weise die Bundesrepublik als Stationierungsland ein spezifisches Mirspracherecht für den Fall eines Einsatzes der Pershing II Raketen oder der Cruise missiles vom BRD-Gebier aus haben wird. Der Abrüscungsbeauftragte des US-Präsidenten, Roscow, wich in einem Spiegelinterview der Frage aus und beconte: „Die Befehlsregelungen für den Einsacz der Pershing II und der Cruise missiles müssen dieselben sein wie bei den gegenwärtigen vorhande-

\footnotetext{
4 Zitier nach Egon Bahr, W/2s wird aus den Deueschen?, 1982, S. 104.

5 Vgl. Der Splegel Nr. 34/1981, S. 21.

6 Vgl. Der Spiegel Nr. is/ig81. S. 19.

7 Vgl. Der Spiegel Nr. 14/1981, 5. 19: SPD.Präsidiumsmieglied H2ns Koschnick soll laue -Soiegel. den Hínw-cis des amerikanischen Vercidigungsministers Weinbergec, die Ausriseung der US-Truppen in der Bundesrepublik - auch mir Neutronenwaffen - liege im Ermessen Washingtons, mit der Bemerkung quirtien haden: - Das sollen die gleich dreimal machen, dann merkt auch hier jeder, daB die Amerikaner uns behandeln wollen wie die Askaris-. Der Spiegel Nr. j6/1981, S. 25.

8 Vgl. die wiederholten Äußcrungen von Egon Bahr u. a. in: Bahr, Was wird aus den Deurschen?. a. a. 0. 5. 105.
} 
nen Gefechrsfeldwaffen. Der Einsatz ist Sache der milirärischen-politischen Entscheidung zum gegebenen Zeitpunkt und im Verlauf des Kampfes, wenn es dann einen geben sollce.".

Die vorliegende Scudie versucht auf beide Fragen (Umfang der US-amerikanischen Stationierungsrechte; bundesrepublikanische Mitspracherechte vor Raketen-Einsarz) Antworten zu geben. Dabei gehen wir an dieser Stelle nicht näher auf die Problematik ein, ob sich für die Bundesrepublik Deutschland aus dem Brüsseler NATO-Beschluß vom 12. 12. 1979 rechuliche Bindungen ergeben. ${ }^{10}$

\section{B. Wer entscheidet über die Stationierung newer atomarer Waffen in der Bundesrepublik?}

I. Stationierungsbefugnisse aus dem sogenannten Generalvertrag vam 26. Mai 1952 in der Fassung vom 23. Okt. 1954 ?

Durch den zunächst konzipierten, jedoch nicht in Kraft getretenen * Vertrag über die Beziehungen zwischen der Bundesrepublik Deutschland und den ,Drei Mächrenc* vorn 26. Mai 1952, den sogenannten Generalvertrag, der auch mDeutschland-Vertrag* genanne wird, solite im Zusammenhang mir dem Projeke über die •Europäische Verteidigungsgemeinschatt das Besatzungsszatur abgelöst werden und die Bunderepublik die *volle Mache über ihre inneren und äußeren Angelegenheiren* erhairen."

Der Vertragstext von 1952 hatte das Wor *Souveränitäta entgegen den deutschen Forderungen absichesvoll vermieden; es solle $\nsim$ der wissenschaftlichen Ausdeurung überlassen (werden), die Kongruenz oder Inkongruenz dieser (Formel) mit dem Souveränicärsbegriff zu kiären «."

Der nach dem Scheitern des Projekrs der "Europäischen Verteidigungsgemeinschafta (am Mehrheirsvotum der französischen Nationalversammiung) auf der Londoner Neun-Mächte-Konferenz im Oktober 1954 dasn revidierte Vertragstext erklärte derngegenüber eindeutig: Die Bundesrepublik hat die volle Macht eines souveränen Staates über ihre inneren und äußeren Angelegenheicen* (Art. I Abs. 2 des Generalvertrages in der Fassung vom 23. 10.1954$)$. $^{3}$

Freilich ist diese Souveränitär der Bundesrepublik auch nach Inkraftureten des Generalvertrages in mehrfacher Hinsicht eingeschränkt; hierauf ist im Folgenden näher

9 VEl. Der Spiegel Nor j0/1981, S. 89.

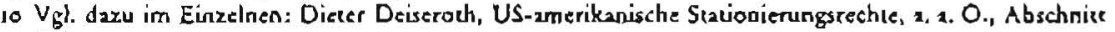
BI.

II Zum Besalzungsstatut vom 10. April 1949 vg!. W. Grewe, Die verfassungsrechulichen Grundlagen der Bundesrepublik Deusschland; Das Besatzungssuatir, Denrsche Reches-Zeieschrif \$ (1949), 5. 265 If.; W. Weagler, Betrachtungen zum Besatzungssuztut, NJW 1949, S. 88 r fí. V scher/Grewe. Bonner Verrag und Zusarzvereinbirunged. München und Rerlin iqgs; vgl. zu den hiscorischen Hineergrinden Kuscher, Seichwore -Bonnee Verrige*, in: Wöreebueb des Völkecrechts 1962 , S. 214 ff.

12 Vgl. Grewc, System und Grundgedanken des Bonner Verrogswerkes. Eipführung zu der erliusercen Textausgabe von H. Kutscher, Bonner Vereag, 19\$2, S. 8: W. Grewe, Von der Kapirulation zum Deutschlandveruag, AuBenpoticik, (19/2), S. 414 fl.

1) Der Vereragsext ist abgedruckt u. 2. bei Ingo von Münch. Dotemence des geteilien Deueschland, 2. Auflage 1976, 5. 129 ff: zu den Hintergrunden dieser Textinderung vgl. u. 2. Wilhelm Grewe, Souveranitat der Bundesrepublik, AoR 80 (1956), 5. 21 If; E. von Putkammer, Vorgesshichee und Zustandekommen der Panser Verrage rom 23. Okx. 1954, Zä̈RV i7 (19/6/57), S. 448 if: aus DDR-Sichs: R. Arzinger, Die Kriegsverrage von Bonn und Paris, Beclin (Osi) 1954: H. Brandweiner. Die Veruräge London und Pans, Neue Jusciz \& (1954), 712 ll. 
cinzugehen, wobei wir uns freilich allein auf dic Frage beschränken, welche Auswirkungen diese Souveränitätsbegrenzungen im Hinblick zuf die geplante Dislozierung der neucn US-arnerikanischen Nuklearsystemc haben.

\section{Vorbehalc des Artikels s Abs. 2 des Generalverrages}

Nach dicser Bestimmung crlöschen ndie von den Drei Mächtea bisher innegehabren oder ausgeübren Rechre in bezug auf den Schutz der Sicherheit von in der Bundesrepublik stationierten Streickräften, die zeitweilig von den Drei Mächten beibehalecn werden ..., sobald die zuständigen deutschen Bebörden entsprechende Vollmachcen durch die deutsche Gesetzgebung erhalten haben und dadurch instandgesetzt sind, wirksame Maßnahmen zum Schutz der Sicherheir dieser Streitkräfte zu treffen, einschließlich der Fähigkeir, einer ernstlichen Störung der öffentlichen Sicherheit und Orónung zu begegnen. Diese sogenannten Vorbehalesrechte rin bezug auf den Schurz der Sicherheita der fremden Strcickräfre in der Bundesrepublik wurden von den westlichen Alliierten im Jahre 1968 explizit für erlosehen erklärt."

Insofern können spezifische Scationierungsrechte gegen den Willen der Organe der Bundesrepublik von den Drei Mächten seir diesem Zeitpunks jedenfalls nicht mehr auf Art. s Abs. $z$ des General- bzw. Deutschlandvertrages gesrützt werden."

\section{Stationierungsvorbehalt in Artikel 4 Abs. I Satz 1 des Generalvertrages}

Nach dieser Bestimmung sollten die Drei Mächte nbis zum Inkrafttresen der Abmachungen über den deutschen Verteidigungsbeitrag ... ihre bisher ausgeübren oder innegehabren Rechee in bezug auf die Scarionierung von Streitkräfecn in der Bundesrepublik behalten. Die »Rechte und Pflichren dieser Streitkräfte sollten sich gem. Arr. 4 Abs. I Satz 3 nach dem sogenannten Truppenverrrag bestimmen. ${ }^{15}$ Mir den in Arrikel ${ }_{4}$ Abs. I Sacz I genanncen Abmachungen über den deueschen Verreidigungsbeitrag* waren der sogenannie "Brüsseler Verrrag" mir seinen Protokullen über die Streickräfre und über die Rüstungskontrolle vom 23. 10. 1954" sowie der Beitritr zum Nato-Vertrag gemeinc." Da alle diese Abmachungen am 6. Mai rgss in Kraft getreten sind," hat dieser in Art. 4 Abs. I Satz 1 Generalvertrag enthaltene Vorbehalt heure keine recheliche Bedeutung mehr. Dies ist in der einschlägigen Fachliteratur unbestritten. Insofern können also Stationierungsrechre auch nicht mehr auf Art. 4 Abs. : Satz 1 Generalvertrag gestützt werden.

\section{Truppenstationierangsrechto aus Artikel \& Abs. 2 Satz 2 des Generalvertrages}

Nach dieser recht komplizierten vertraglichen Bescimmung ist odie Bundesrepublik damit einverstanden, daß vom Inkraftrreten der Abmachungen über den deurschen

$14 \mathrm{Vgl}$. die Bekannemachuag vom 18.6. 1968. BGBI. I S. 714: zu den historischen Hìncengründen vgl. Dierer Sternel, Kricik dee Noustandsegescize, 1968, S. 14 f., 26 ff.

is Ebenso: Helmuc Rumpi. Land ohnc Souveränicsit. 1. Auil. 1973. 5. 19; Meinhard Sthróder. Die Auswirkungen der Nolstandverlassung auf dic Souveranitz̈t der Bundesrepublik. Europa-Archiv 1968. 5. 78 ff: sowie jüngse: Diubler, Stationicrung und Grundgesecz, S. 8 ; $\mathrm{H}$. Ridder. Dic Souverinicà der BRD und ihre Position in der westlichen Allianz, BlfdelneP 1982. Heft 8 .

16 Der - Vercrag über die Rechee und Pflichten ausländischer Sureiskrafue und ihrer Mirglieder in der Bundesrepublik Deueschland., der sogenannie Truppenverersg, ist abgedruekt in: BGBI. s95s II, S. 121 . 17 BCBI. 1915 II. S. 28 \}.

18 So zu Reche auch Rumpi, Das Reche der Truppenseztionierung in der Bundesrepublik. 1969. 5. 7

19 Vgl. das Zustimmungageserz zu diesen Verragen rom 24- 3. 195s. BGBI, II. S. 196 und die Bekannemachung rom 9. \{. 1951 uber itre Inkrafureten in BGBI. II. 610. 
Verteidigungsbeitrag an Streitkräftc der gleichen Nationalität und Elfekrivsrärke wie z. Zt. dieses Inkrafteretens in der Bundesrepublik stationiert werden dürfen «. Weiter heißc es im Satz 3 der Vorschrift: "Im Hinblick auf die in Art. I Abs. 2 dieses Vertrages umschriebene Rechrsstellung der Bundesrepublik und im Hinblick darauf, daß die Drei Mächte gewille sind, ihre Rechte betreffend die Starionierung von Streickräften in der Bundesrcpublik, soweit diese betroffen ist, nur in vollem Einvernehmen mic der Bundesrepublik suszuüben a, sollte die "Frage der Truppenstationierung in einem besonderen Vercrag geregelt werden $\alpha$ (Arr, 4 Abs. 2 Satz 3 Generalverrrag). Diese besondere Regelung erfolgte im sogenannten Aufenchalrsvertrag vom 23. 10. 1954, der zusammen mir dem Generalvertrag in Kraft trat. ${ }^{20}$ Der Zrveck der Truppenstacionierung wird im Vorspruch dieses Aufenthaltsvertrages mit der „gegenwärrigen internacionalen Lage und mic der Notwendigkeir begründer, "die Verteidigung der freien Wels sicherzustellen *; diese Gründe erforderten *weirerhin die Anwesenheit ausländischer Streickräfre in der Bundesrepubliku.

In Art. 1 Abs. $\mathrm{t}$ dieses Aufenthaltsvertrages wird der im Generalvertrag in Art. 4 Abs. 2 Satz 2 enthaltene Grundsacz bekräftigt, daß die Bundesrepublik damic einverstanden sei, "daß vom Inkraftreten der Abmachungen über den deutschen Verteidigungsbeitrag an Streickräfte der gleichen Narionalicät und Effektivscärke wie z. Zr. dieses Inkrafturetens in der Bundesrepublik stationiert werden dürfen 4 . Sollen zukünftig zusätzliche Streickräfre der Partnerstaaten des Aufenchaltsvertrages (USA, Großbritannien, Frankreich, Niederlande, Belgien, Kanada) im Bundesgebier üben oder soll die Effekuvscärke der Streitkräfe erhöht werden, so bedürfen diese Veränderungen und Bewegungen der Zustimmung der Bundesregierung; Übungen müssen mit den Richclinien der Nato übereinscimmen; die übenden Truppen dürfen nicht länger als 30 Tage in Bundesgebiet verweilen (Arr. r Abs. 2 u. 3 des Aufenthaltsvertrages). Außerdem gewährte die Bundesrepublik den amerikanischen, britischen und französischen Streickräfren ein Durcbzugsrecht auf dem W/ege in andere Mirgliedsstaaten des NATO-Vertrages auf der gleichen Grundlage, wie dies zwischen anderen NATO-Mirgliedsstancen üblich isc, oder mic Wirkung für alle Mitglieder im NATO-Rat vereinbart wird (Arc. I Abs. 4 des Aufenthalesvertrages). Der Aufenthaltsvertrag stritt außer Kraft mit dem Abschluß einer friedensvertraglichen Regelung mic Deutschland oder wenn die Unzerzeichnerstazzen zu einem früheren Zeitpunkt übereinkommen, daß die Entwicklung der iaternationalen Lage neue Abmachungen rechufertigi $\alpha$ (Art. 3 Abs. I).

Ohne das wvolle Einvernehmen mir der Bundesrepubliks, also ohne Zustimmung der zuständigen Organe der Bundesrepublik, könnte somit also die Dislozierung der im Nato-Doppelbeschluß genannten amerikanischen atomaren Waffensysteme gem. Arc. 4 Abs. 2 Sacz 2 u. 3 in Verbindung mic Arr. I des Aufenthaltsvertrages nur dann erfolgen, wenn diese neuartige Ausrüstung der amerikanischen Streitkräfte yon dem seinerzeit erklärten Einverständnis mit der Stationierung von Streitłräften *der gleichen Nationalität und Effektivstärkea (wie am 5. 5. 1955) gedeckt würde. Die *Effekcivstärkes am s. bzw. 6. 5. 195s wurde niemals amelich bekanntgemacht."

Juristisch gesehen hängt die Beantwortung dieser Frage davon $a b$, ob die Einführung der neuen nuklearen Mitrelstreckensysteme bei den in der Bundestepublik stationierten US-Sereitkräfren die damalige (195s) „Effektivstärke veränder oder nichc.

20 Verrag uber den Auienthalı auslindischer Strcirteräle in der Bundesrepublik vom 23. 10. 1954 (. Autenthalisverirag.), BGBI, 1955 (II, S. 293.

2) So auth Hiensrdorfer, Fachwiss. Diensr des DL Bundesiages, 1981. S. s; zu den Truppenseirken im Jahre 198 ! vgl. die Broschuce des Presse- und Informationsamies der Bundesregierung - Die all. Sucionierungsstreitkräle in der BRD., August iy91. 
Würde die *Effckrivstärke* durch diese neuen Svsteme erhöht, könnte die Stationierung nur im Einvernehmen mir den zuständigen Organen der Bundesrepublik erfolgen.

Würde man den Begriff der Effekcivstärke nur auf die Truppenstärke, also auf die Anzahl der Truppenangehörigen beziehen, wäre die - Bewaffnunga dieser Streitkräfte nicht von den oben zitierten Bestimmungen erfaßt. Allerdings ist in der fraglichen Bestimmung eben von "Effektivstärke" die Rede; dennoch ist der Wortuut der Vorschrift in dieser Hinsiche nicht eindeurig. Ein Vergleich dieser Begrifflichkciten mit gleichlautenden oder ähnlichen in anderen vertraglichen Regelungen (auBerhalb des Generalvertrages und des Aufenthaltsvertrages), z. B. mit einzelnen Regelungen des $*$ Brüsseler Vertrages (BGBI. 1955 II, S. 256, 5. 262, S. 630), ist schon deshalb nicht sinnvoll, weil es sich dabei eben um andere Verträge mic anderen Vertragspartnem bandelc.

Von Bedeurung für die Auslegung ist allerdings die Tarsache, daß jede Truppenstacionierung zwangsläufig einen Eingriff in die Souveränität jedes •Aufnahmestaates * darstellt.

Da der Bundesrepublik durch Art. I Abs. 2 des Generalvertrages, wie oben bereits dargelegt, „die volle Macht eines souveränen Staatcs über ihre inneren und äußeren Angelegenheiten a eingeräumc wurde, gilt dies zumindest nach Inkraftcreten des Generalvertrages auch im Hinblick auf die Souveränitär der Bundesrepublik.

Gerade weil sowohl die Bundesrepublik als auch die USA (cbenso wie Großbritannien und Frankreich) Mitglieder der Vcreinten Nacionen sind, haben sie zudem seit dem Beginn ihrer Mitgliedschaft auch die Pflicht, die Verpflichrungen aus der Charta der Vereincen Nationen zu erfüllen; die Organisation der Vereinten Nationen beruht nun aber auf dem Grundsacz der souveränen Gleichheic aller ihrer Mirglieder (Ar. 2 Nr. I der Charta), in deren innere Angelegenheiten selbst die Organisacion der Vereinten Nationen grundsärzlich niche eingreifen darf (Art. 2 Nr. 7 der Charta). Daraus läßt sich mir guten Gründen die Auslegungsmaxime ableiten, daß zwischen den Staaten geschlossene völkerrecheliche Verträge im Zweifel so zu verstehen sind, daß sie die Souveränirär der Vertragspartner möglichst wenig einschränken. ${ }^{11}$

Bereics dies würde zu dem Ergebnis führen, daß die Dislozierung neuer amerikanischer aromarer Waffensysteme bei den bereits stacionierten Streitkräften eine Veränderung der Effektivstärkea bedeutet. Für diese Auslegung spricht im übrigen auch der sehr langwierige und auf hoher politischer Ebene geführte Entscheidungsprozeß, der zum NATO-Doppelbeschluß führe. Gerade wenn der Beschluß offiziell als »Nachrüstungsprogramm * und damit als Projekr zum Ausgleich einer (vorgeblich) höheren Effektivstärke der Streitkräfce des Warschauer Pakces begründet wurde und wird, wird damit eben eine Erhöhung der *Effekrivstärkex der US-bzw. NATO-Streitkräfre in Westeuropa angezielt. Angesichts der rasanten Eskalation des Abschreckungs- und Kriegsführungspocencials der US-Streitkräfce in Westeuroga gegenüber der Situation im Jahre rgss von einer øgleichen Effektivstärke « wie z. $Z_{\text {r. }}$. des wInkrafturetensa des Generalvertrages am s. S. Ss zu sprechen, wäre geradezu riefstaplerisch.

Insofern kann einer vom Bundesverteidigungsmínisterium publizierten Stellungnahme zur Frage des Zuscimmungsvorbehaites bei der Stationierung neuer Waffen uneingeschränkt zugestimmt werden, in der es heißc:

21 Im Ergebnis ebenso z. 8. Düubler. Stazionienng und Grundgeseez. 1982, S. 104 unter Berulung aut Georg Dahm. Völkerrechu, Band 1, Stutegar 1958. 5. 168: Paul Guggenheim. Lehrbuch des Völkerreches. Band , Basel 1948, S. 128 mit weiteren Nachweisen. 
-insbesondere wird auf cine Zustimmung in den Fällen niche verzichcet werden können, in denen diese neuen Entwicklungen über den militarischen Bereich hinaus eime Bedeutung bekommen haben, die von enrscheidendem Einflub auf die innen-, auBen- und sicherheirspolicische Handlungsfähigkeit des Auinahmestaates ist. Wären derarı gravierende Vorhaben der Einflußnahme des Aulenthaltsstzates enrzogen, so wurde seine Souveränitär in unzulässiger Weise becinträchrigt 4 . Referat VR II 4 - AZ: $10-$ s - os - 01, (liegr dem Verfasser vor).

In der gleichen Verlaurbarung zitiert das Vereidigungsministerium zudem einen Beschluß der NATO-Razs-Tagung vom 16. bis 19. Dez. 1957 in Paris, in dem es hinsichtlich der Verteilung an Vorräcen von Acomsprengköpfen heißs: "Die Verceilung dieser Vorräte ... und die Vereinbarang für ihre Verwendung werden infolgedessen in Übereinstimmung mir den Verteidigungsplänen der NATO und im Einvemebmen mit den direkt beteiligten Staaten festgelegt ......'s

- Einvernehmena seczr nun aber Zuscimmung der Bereiligren voraus. Zwar ist im Völkerreche die Bindungswirkung einer Auslegung umstrirten, die sich in einer gleichartigen nachfolgenden Praxis aller vertragsschließenden Parreien ausdrückt." Diese Praxis gewinnt jedoch jedenfalls dann ihre gewichcige Bedeutung, wenn sie allein der völkerrechtlich normierten »souveränen Gleichheit» aller Staaten, zumal der UN-Mitgliedsstazien, gerecht wird.

4. Stationierungs- bzw. Dislozierungsrechce awfgrund des Artikels 4 Abs. 2 Satz I Generalvertrag in Verbindung mit Artikel 2 Generalvertrag?

Artikel 4 Abs. a Sarz I Generalvertrag besagt, daß die svon den Drei Mächten bisher ausgeübten oder innegehabcen und weiterhin beizubebaltenden Rechte in bezug auf die Stationierung von Streickräfren in Deutschland. . . von den Bestimmungen dieses Arrikels nicht berührt werden. Die damit angesprochenen weiterhin beizubehal-

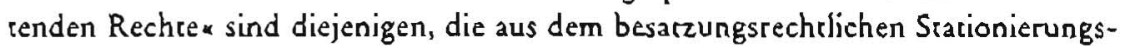
reche resultieren.

Jedoch sollen nacb eben dieser Bestimmung (Arikel 4 Abs. 2 Sarz i Generalvertrag) diese Rechte nicht im bisherigen, sondern nur in einem beschränkten Umfange weirergelten (msoweit sie für die Ausübung der im ersten Sarz des Arrikels 2 dieses Verrages genannten Rechte erforderlich sind $\alpha$; Artikel 4 Abs. 2 Sacz I Generalver(rag). Die »Erforderlichkeit der konkreten Beibehaltung dieser Rechte wird also durch Arrikel 2 des Generalvertrages bestimmr; danach behalten die Drei Mächte -im Hinblick auf die intemationale Lage, die bisher die Wiedervereinigung Deurschlands und den Abschluß̉ eines Friedensvertrages verhindert hat, . . die bisher von ihnen ausgeübten oder innegehabten Rechte und Verantwortlichkeicen in bezug auf Berlin und auf Deutschland als Ganzes einschließlich der Wiedervereini gung Deutschlands und einer friedensvertraglichen Regelunga."s

Bei diesem westalliierten Vorbehalt der bisherigen ${ }^{2}$ Rechte und Verantwortlichkeiten \& handelt es sich also um eine Ausklammerung von bescimmten rechtlichen Befugrissen aus dem Vertrag; die Übertragung der Souveränität an die Bundesrepublik Deurschland sollte sich nicht auf die in Ar. 2 den Westmächten vorbehalcenen Gebiete beziehen. Nach dem Wortlaut der Bestimmung haben die Westalliierten die reservierten Rechre bebalten, also nicht vertraglich erhalten. Für diese Ausicgung spriche auch, daß Arr. 9 des Generaivertrages, der in seinen ersten beiden Absätzen ein Schiedsgericht zur Entscheidung für alle Streirigkeiten einsetzt, die sich aus dem

2) Der Beschluß ist abgedruckt in: Archiv der Gegenware v. 19. 12. 57.5 .6802 ff (Ziff. 21).

24 Vgl. R. Lagoni in: Menzel/losen, Volkereche 3. Aull, 1979. S. $117 \mathrm{~m}$. w. N.

is Vgl. den umelichen Texr bei von Minch, 1. 2O., S. 1 jo. 
Vercrag ergeben, im dritcen Absatz die Screicigkeicen aus Arrikel 2 von dieser Schiedsgerichtbarkeit ausnimme. Dies deutet zumindest darauf hin, daß das. was in Arcikel 2 geregelt worden ist, nicht als Vertragsgegenstand betrachect wird. ${ }^{16}$

In der Begründung der Bundesregierung zu Arrikel 2 des Generalverrages hieß cs, daß sich diese "Rechte und Verantwortlichkciten nauf die Vereinbarungen von i94s gründen $\alpha .{ }^{27}$

Im Bundestagsausschuß für Auswärtige Angelegenheiten war seinerzeit bereits darüber diskutiert worden, nauf welchen internationalen Vereinbarungen die beizubehaltenden Rechte der drei wcstlichen Mächte beruhen. Eine Einigkeit darüber, ab nur die Deklaration vorn s. Juni 1945 oder daneben auch das Porsdamer Abkommen oder nur dieses in Berrache kumune, war richr zu erzielen a."

Auch in der Literatur isc eine präzise Rechrsgrundlage für die seinerzeitigen Besaczungsrechre nichr eindeutig auszumachen; zumeist wird auf die occupatio bellica im Sinne der Haager Landkricgsordnung (HLKO), aul die Übernahme der Obersten Regierungsgewalt durch die Siegermächte am 5 . Juni 1945 oder auch auf die bedingungslose milizärische und politische Kapiculation Deucschlands am 8. 5. 1945 abgcstellt..29

Allgemein anerkannt ist jedoch, daß die Rechte der Siegermächre jedenfalls in der sogenannten Berliner Erklärung vom \&. Juni 194 s näher bestimmt wurden, in der es hieß:

"Dic Regierungen des Vereinigten Königreichs, der Vereinigten Stazren von Amerika, der Union der Sozialistischen Sowjetrepubliken und die Provisorische Regierung der Franzosischen Republik übernehmen die oberste Regierungsgewalk in Deutschland einschlicßlich aller Befugnisse der Deutschen Regierung, des Oberkommandos der Wehrmacht und der Regierungen, Verazlungen oder Behörden der Länder, Srädte und Gemeinden-..30

Innerhaib ihrer Besarzungszonen hatten die drei Westmächre im Zuge der von ihnen geförderten Bildung eines westdeurschen Staates den westdeutschen Behörden zunehmend hoheitliche Befugnisse übertragen. Exappen auf diesem Wege waren unter anderem das am 10. April 1949 geschlossene und an 21. September 1949 in Kraft getretene Besatzungsstatut sowje das Petersberg-Abkommen vom 22. November 1949 und der sogenannte Überleitungsvertrag vom 26. Mai igs2."

Bekannclich haben dic Drci Mächre - abgësehen von den im Rahmen dieses Abschniztes untersuchcen. Vorbehalusrechten - in Art. I des Generalvertrages ausdrücklich erklärt, daßs «mit dem Ink rafttreten « des Generalvertrages ndie Vereinigten Staaten von Amerika, das Vereinigte Königreich von Großbritannien und Nord-Irland und die Französische Republik ... das Besatzungsregime in der Bundesrepublik

26 Vgl. dazu auch Oto Kimminich, Die Souveränitär der Bundesrepublik Deutschland, 1970, S. 87.

$27 \mathrm{Vgl}$. Regierungsbegründung zum Geselzentwurf becreffend das Protokall vom 2\}. Okt. 1994 über die Beendigung des Besatzungsregimes in der Bundesrepublik. Deutscher Bundessag. Drucksachen. Nr. 1000 , 2. Wahlperiode. S. 38.

$28 \mathrm{Vgl}$. den Schrítlichen Berichr des Ausschusses für Auswänige Angelegenheiten über den Enımuri eines Gesetzes betreffend das Protokoll vom 23 . Okt. 1954 über die Beendigung des Besazzungseegimes in dec Bundesrepublik Deutschland. Deurscher Bundestag, Drucksachen Nr. 1200, 1. IVahlperiode. S. 6.

19 Vgl. dazu u. 1. Otro Kimminich, Die Souveränicit der Bundesrepublik Deuseschland, 2. 1. O.. S. ja ff: Klaus Freiherr von Richthofen, Die Wiedererlangung der deuschen Souveranität für das Gebiet der Bundesrepublik Deuischland nach den Pariser Venrägen vom 23. Okt. 1954. Dissertacion Göctingen 197:, S. 58 ff: Osto Großmmnn. Aufesthalesrecht und Rechtssteilung der auslïndischen Strcitkräte in Deutschland unter besonderer ürdigung der Rechuslage der Französischen Truppen. Disserracion Wurzburg 1971. 5. 6 (I); Rumpf, Land ohne Souveränitat, a a. O., S. 18: Adoll Amdt, Der deutsche Stast als Rechisproblem. Berlin ig\%o. 5. 27; Ulrich Seheuner. Beitriat der Bundesrepublik zur curopaischen Verreidigungsgemeinschaff und Grundgesctz, in: Der Kampf um den Wishrbettrag, zwetter Halbband München 1953, 5. 9411,149 .

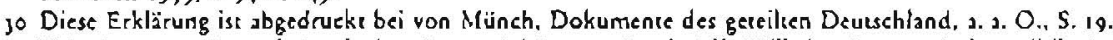

II Vgl. dazu u. a. Rumpr, Land ohne Sauveräniẗ̈, 1. A. O. S. 8 If: Wilhelm Grewe, Außenpolitik der

Nachkriegszeit, Sruzegart 1960. 
beenden, das Besatzungsstatut autheben und die Alluerte Hohe Kommission sowie die Dienststellen der Landeskommissare in der Bundesrepublik auflösen.."

Damir haben die Drei Mächte also ausdrücklich suf ihre spezifischen Rechre verzichter, die sie als Drei Mächce im Rahmen des . Besatzungsregimes in der Bundesrepublik « inneharten. Geblieben sind nach dem Wortlaur des Arrikels 2 des Generalveruages lediglich die Rechte in bezug auf Berlin und auf Deutschland als Ganzes einschließlich der Wiedervereinigung Deutschlands und einer friedensvertraglichen Regelung*, die aber den Drei Mächten nicht allein, sondern als drei der »Vier Mächrě zustehen. Diese Auffassung ist auch von den Regierungen der Drei-WestMächte in gleichlautenden Noten vom I1. August 1970 anläßlich des bevorstehenden Abschlusses des deutsch-sowjetischen Vertrages vom I2. August 1970 bestärigr worden. Darin hieß es, $\nsim$ daß die Rechte und Verantwortlichkeiten der Vier-Mächte in bezug auf Berlin und Deurschland als Ganzes, die sich aus dem Ergebnis des 2. Welk rieges herieiren und die im Londoner Übereinkommen vom r4. Nov. 1944, in der Vierer-Erklärung vom s. Juni i 94 s sowie in anderen Kriegs- und Nachkriegsübereinkünften ihren Nicderschlag gefunden haben, durch einen zweiseitigea Vertrag zwischen der Bundesrepublik Deutschland und der Union der sozialistischen Sowjerrepubliken, einschließlich dieses Vertrages, nicht berühn werden und nicht berührt werden können. «

Auch in dem "Vier-Mächre-Abkommen über Berlin" vom 3. Sepr 1971 haben die Regierungen Frankreichs, der Sowjecunion, Großbrizanniens und der USA erklärt, sie handelten wauf der Grundlage ihrer Vier-Mächte-Rechre und -Verancworclichkeiren und der entsprechenden Vereinbarungen und Beschlüsse der Vier Mächte aus der Kriegs- und Nachkriegszeir, die nicht berührt werden . $^{\text {H4 }}$

- Rechre und Verantwortichkeiten in bezug auf Berlin und auf Deurschland als Ganzes einschließlich der Wiedervereinigung Deutschlands und einer friedensvertraglichen Regelung*, wie sie in Arrikel 2 des Generalvertrages erwähnt sind, können daher allenfalls von den Vier Mächten ausgeübt werden; die Vereinigren Staaten etra könnten diese Rechre keinesfalls alleine ausüben. Dieses würde auch für eine eventuelle Stationierung neuer amerikanischer Mittelscreckensysteme gelten: Weder die US-Regierung noch die Regierungen der anderen früheren Besarzungsmächce könnten alleine unter Berufung auf die Besaczungsrechte Besatzungsgewalt ausüben.

Könnte man - so bliebe zu fragen - dessen ungeachtet aus der Tatsache, daß seit der Beendigung der Täugkeit des *Allïerzen Kontrollrates* im Jahre 1948 jede Besatzungsmacht innerhalb ihrer Besatzungszone die Besatzungsrechte allein ausgeübr hat, folgern, der in Artikel 2 des Generalvertrages festgehaltene Vorbehalt der wausgeübcen oder innegehabren Rechce und Verantworclichkeitene gewähre weizerhin ein besatzungsrechlich fundiertes Truppenstacionienungsrecht jeder einzelnen (früheren) Besatzungsmacht? Würde man diese Frage bejahen, wären damit die Inhaber eines solchen (besarzungsrechrlich verankerten) Truppenstationierungsrechres von

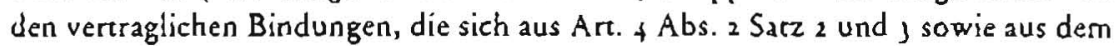
Aufenthaltsvertrag (siehe oben) ergeben, u. U. freigesiellt: Die Rechte auf Truppenstationierung könnten dann ungeachter der in jener Bestimmung genanncen Grenze der "gleichen ... Effekrivstärke* wahrgenommen werden.

Die Bundesregierung hatte bei der parlamentarischen Beratung der Zustimniungsge-

$12 V_{G} l$ den Text bei van Münch, Dokumente des geceilen Deutschland, 2 2. O, S. a jo,

31 V Vl. den Texe der amerikaniscben Nore in: Presse- und Informationsamt der Bundesregierung (Herausgeber), Die Veruáge der Bundesrequblik Deuesciland mis der Unon der sozi aliscischen Sowjetrepubli. ken vom 12. August igyo und mit der Volksrepublik Polen vom 7. Dez. rayo. S. is fil.

14 Vgl. Presse- und Infonmacionsame der Bundesregienung (Herausgeber). Das Vier-iMächte-Abkommen uber Berlin rom 3. Sept. 1971. S. is. 
setze dic Auffassung vertreten, es bestünde seit dem Abschluß des Generalvertrages und des Aufenthaltsvertrages für das Gebier der Bundesrepublik nur noch ein einheirliches vertragliches Stazionierungstecht. Die Rechte der Drei-Mächee ergäben sich insoweir ausschließlich aus der im Generalvertrag und im Aufenchalusvertrag freiwillig übernommenen Verpflichtung der Bundesrepublik."

Auch Grewe, der an den Verhandlungen mir den West-Alliierten maßgeblich bereiligt war, vertrat die Auffassung, nach Abschluß der Verträge sei das besatzungsrechtliche Stationierungsreche nicht nur sachlich (durch die Bindung seiner Ausübung in der Bundesrepublik an deren Zustimmung), sondern auch territorial beschränkr worden; für das Gebict der Bundesrepublik existiere es nicht mehr. Dor sci es vielmehr durch ein vertragliches Stationierungsrecht ersetzt worden; wein auf Besatzungsrecht gegründetes Stacionierungsrecht der West-Mächte nach dem Inkrafttreten der Abmachungen über den deutschen Verteidigungsbeitrag (bestehe) nur noch in Berlin - und (seinerzeit) im Saargebieca. ${ }^{36}$

Demgegenüber zieht etwa Rumpt aus der Vorbehaltsregelung in Artikel 4 Abs. 2 Satz $x$ in Verbindung mit Artikel 2 des Generalvertrages den Schluß, daß, nsowcic diese Truppen der gemeinsamen Vereidigung dienen, ... ihre Anwesenheit auf Vertrag (beruht); soweit sie der Durchsetzung der Rechte der Drei Mächte auf Mirbestimmung bei der Wiederherstellung der Deutschen Einheit dienen, ist die ursprüngliche besatzungsrecheliche Grundlage erhalten geblieben «."

Freilich ist, wenn man sich die Auffassung von Rumpl und Hienstorfer zu eigen machr, unklar, wonach sich bemißt, ob die Strcitkräfte der West-Alliierten jewreils als Truppen zur Verceidigung der freien Welt (Aufenchaltsvertrag bzw. Art. 4 Abs. 2 Satz 2 u. 3 Generalvertrag) oder aber ob sie sin bezug auf Berlin und auf Deurschland als Ganzes einschließlich der Wiedervereinigung Deurschlands und einer friedensvercraglichen Regelung agieren.

Auch im anglo-amerikanischen Rechtsbereich wird cine solche Aufteilung derseiben Starionierung in eine isolierte besatzungsrechtiche und eine isolierte vertragliche Grundlage abgelehnt. Bathurst und Simpson etwa gehen davon aus, daß die Rechte der Drei-Mächte unteilbare Rechte sind, welche nicht einerseits aufgegeben und andererseits für andere Zwecke aufgehoben werden könnten; sie vertreten die AufCassung, bescimmte Rechte seicn für unbestimmte Zeit den Drei West-Mächten vorbehaiten geblieben, obgleich die Bundesrepublik den Status eines souveränen Staates besäße; zu den Rechten gehöre die Stationierung von Streitkräften in Deutschland ${ }^{38}$ Unablängig davon, ob nun das Truppenstationierungsrecht immernoch eine besatzungsrechtliche Wurzel hat," könnte jedenfalls die Dislozierung der neven amerikanischen nuklearen Waffensysteme nur dann auf Artike 4 Abs. 2 Sarz I i. V. mit Arrikel 2 Satz r des Generalvertrages gestürzt werden, wenn gerade dadurch die Drei-Mächce einen Beitrag zur w Wiedervereinigung Deutschlands und einer friedensvertraglichen Regelungw anstreben bzw. Jeisten wollten. Eine solche Annahme ist schon deshalb absurd, da bisher weder die Bundesregierung noch die US-Regierung dic Auffassung venteren haben, bei der Stationierung der Pershing II -Rakecen

15 So auch der Generalberichtersurter der Bundestagsausschüsse, der Abgeordnete Furler, Sitzungsbericht des 2. Deutsehen Bundestages, 69. Sitzung vorn 24. Febr. 195s, S. 3516, 3524 .

$36 \mathrm{Vgl}$. Wilhelm Grewe. Souveränitä̀ der Pundesrepublik. AöR 80 (195/5/56), S. 211 ff, 218 ff.

37 Vgi. Rumpf. Das Reche der Truppenstacionierung in der Bundestepublik, 1969, S. 8; dieser Auffassung schließr sich auch Hienstorfer (Wissenschafrlicher Fachdienst des Deutschen Bundestages) in sennem Gutacheen vom 30. 9. 1981. 5. 3, an.

18 Vgl. M. E. BathursuJ. L. Simpson. Germany and the Norch Atlancic Community, London 1956, 5. 137: vgl. ferner ). Bishop. The Concractual Agreements with the Federal Republic of Germany, in: American Journal of Inernauonal Law 1953. S. 147.

19 kritiset hierzu jungst auch Ridder. Die Souverinitat der BRD und the Posicion in der westlichen Allizan. Ig81, S. 10 l im Manuskript. 
und der Marschllugkörper gehe es um die Ausübung der Vorbehaltsrechre nach Artikel 2 des Generalvertages. Die bisherige Rechtfertigung stützte sich auf vorgebliche oder catsächliche Disparitáten militärischer Art gegenüber den Streitkrälten des Warschauer Pakces. Ein Zusammenhang mit den Rechten aus Artikel z des Generalvertrages ist auch schlechterdings nichr ersichtlich. ${ }^{\circ} \mathrm{W}$ ir können darnit festhalten, daß ungeachter der juristisch z. T. umstritcenen Zweifelsfragen über den Inhalt des Vorbehaltsrechtes aus Artikel 4 Abs. 2 Satz 1 in Verb. mir Arrikel 2 Sacz 1 des Generalvertrages eine Dislozierung der neuen amerikanischen W/affensysreme (gegen den Wilien der zuständigen Organe der Bundesrepublik) niche auf diese Bestimmungen gescützt werden kann. Der Frage, ob aus dem NATO-Truppenstatur und den damit in Verbindung stehenden Verträgen und aus dem sogenannten Brüsseler Vertrag Stationierungsrechte folgen, gehen wir - weil die Problemarik relariv unstreitig ist - hier nicht näher nach."

\section{Siationierungsrechie aus Geheimabkommen?}

Unsere bisherige Untersuchung hat uns zu dem Ergebnis geführt, daß eine Stationierung neuer US-amerikanischer atomarer Mittelstreckenwaffen in der Bundesrepublik aufgrund der untersuchten Abkommen und Vereinbarungen ohne Zustimmung der zuständigen Organe der Bundesrepublik rechtlich nicht zulässig wäre.

Wir hatren freilich eingangs bereits Presseberichte ziciert, wonach Vertreter der USRegierung behauprer haben sollen, es gäbe Gebeim-Abkommen, die die Starionierung neuer Atomwaffen lediglich von Konsultationen, nichr jedoch aber von $\mathrm{Zu}-$ srimmungen der zuständigen Organe der Bundesrepublik abhängig machten.

Aus Pressemitceilungen ist auch bekannt, daß in den Jahren jedenfalls zwischen 1974 und 1977 eine Vereinbarung abgeschlossen worden sein soll, deren genaue Bezeichnung lauret: "Abkommen zwischen der Regierung der Bundesrepublik Deurschland und dem Obersten Alliierten Befehlshaber Europa über die Aufreilung der Zuständigkeicen sowie die Zusammenarbeit der Nato-Befehlshaber und der territorialen Befehlshaber im Kriegeu. Dieses Abkommen wurde nie veröffenclichr; bekannt wurde jedoch, daß lür die Bundesrepublik Staatssekrerär Walter Gehlhoff vom Auswärtigen Amr und General Harald Wusr, Generalinspekreur der Bundeswehr, für die Nato der (damalige) Oberste Alliierte Befehlshaber General Alexander Haig unterzeichneten; die Tagespresse meldete seinerzeir, dem Naco-Oberbef́ehlsbaber SACEUR würden dadurch weitgehende Rechre auf deutschem Boden im Krisenfalle eingeräume."

Ferner soll es den Entwurf eines Regienungsabkommens über den Status der USTruppen im Alarmzustand geben; danach sollen ndie US-Streitkräfre das Recht (haben), jedes Gebiet und jede Einrichnng (bei Alarmzustand) zu beserzen, die sie für die Erfüllung ihrer Aufgaben oder für ihre Sicherheit gemäß den Entscheidungen der Befehlshaber für nörig hakicen, und dort solches Gerät $z$ installieren, das sie für diese Zwecke für nölig halcen a. Für den Fall innerer Unruhen, die den Aufrrag oder die Sicherheit der US-Streitkräfre beeinträchtigen könncen, wie z. B. die Anwendung von Waffengewalt oder um sich greifender Aufruhr, soll sich die Regierung des Aufnahnelandes verpllichrer haben, ssolche Unruhen mit den ihe zur Verfügung

40 Ebenso jüngse: Däubler, Stavionterung und Grundgeserz, S. 107.

$\$ \mathrm{Vgl}$. dazu Dieter Deisecoch, US-amerikanische Sravionienungsrechte, s. 2. O., Abschnut B III und IV.

i2 Vgl. Suddeursche Zeitung rom 10. 2. 1977 und vorn 21. 2. 1977. 
stchenden Mirteln zu unterdrücken. Sollecn sich dicse Maßnahmen jedoch als unwirksam erweisen, oder die Regienng von ... um Uncerstüczung bitcen oder der US-Befehlshaber der Meinung sein, daß die Regierung von ... nicht die Fähigkeit besitzr, solche Unruhen wirksam oder rechtzeitig zu unterdrücken, dann können die US-Streitkräfte Maßnahmen treffen, die der US-Befehlshaber für nörig hälı.

*Weiterhin können nach diesern Abkommen die US-Streirkräfte solche militärischen Operationen durchführen, die erforderlich sind . Die Regierung des Aufnahmclandes (BRD) ermä̀chrigr die US-Streitkräfte, - Kernwaffen (in die Bundesrepublik) zu bringen (und) sie dort zu stationieren, wo es für nötig gehalten wird a."s

Ob beide Abkommen wirklich abgeschlossen worden sind, welchen genauen Inhalt sie haben und ob darüber hinaus ähnliche oder ergänzende (Geheim-) Abkommen existieren, entzieht sich (naturgemäß) unserer Kenntnis. Wir wollen uns jedoch im Folgenden der Frage widmen, ob diese (Geheim-) Abkommen u. U. recheliche Wirkungen entfalten können.

Bis zum Ende des ersten Welckrieges waren Geheimverträge und geheime Zusatzabkommen ein viel verwandtes Instrument der Außenpolitik. Das seinerzeir gelrende Völkerrecht machre die Gültigkeit solcher Vereinbarungen niche von der Publikacion abhängig; es kannte auch keine speziellen Normen für Geheimverträge oder Geheimarrikel; vielmehr wurden diese Vereinbarungen wie alle anderen völkerrechtlichen Abkommen behandelt. Im Anschluß an die Forderung im ersten der sogenannten *vierzehn Punkte des amerikanischen Präsidenten Wilson aus dem Jahre 19 r 8 wurde dann erstmals in Arikel i 8 Satz 2 der Sazzung des Völkerbundes eine völkerrechcliche ${ }^{2}$ Registrierungspflicht* normiert; in Zukunft sollten alle internacionalen Verträge und A bmachungen beim Sekretariar des Völkerbundes eingetragen und möglichst bald veröffentlicht werden; kein Venrag solle vor dieser Eintragung rechererbindlich sein."

Die Tragweite des Arcikels 18 Abs. 2 der Völkerbundsatzung war von Anfang an umstritten; zudem wurde die Registrierungspflicht in der Folgezeit *teils aus Nachlässigkeit, teils bewußt nicht stecs beachcec. as In der Saczung der Vereinten Nationen wurde versuche, diesen Erfahrungen Rechnung zu tragen. Artikel 102 der Charta der Vereinten Nationen machte deshalb die Registrierung nicht mehr zur Bedingung der Gürtigkeit von Staatsverträgen, sondern verpflichtete die Mirgliedsstaaten, ihre in kraft getretenen Staatsverträge zu registrieren; bei einem Unterlassen dieser Registrierung können sich nach Arrikel 102 Abs. 2 die Verragsparteien bei einem Organ der Vereinten Nationen nicht auł solche Verträge und internacionale Übereinkünfte berufen. Diese Bestimmung verbieter also den Abschluß von Geheimverträgen nicht, sondern bedrohr lediglich die in nicht registrierten Verrägen erzeugten Normen »mit relaciver Unwirksamkeic, nämlich bezüglich der Durchseczbarkeit vor Organen der Vereinten Nationen $\alpha^{.60}$

Artikel 102 erfaBt alle völkerrechtlichen Vereinbarungen. Die Form und Bezeichnung der Vereinbarung, die Narur, Bedeutung und Dauer der Verpflichcung sind . . . unerheblich "; ausgeschlossen sind lediglich Verträge von Völkerrechtssubjekren mit Nichr-Völkerrechessubjekten oder mir solchen partiellen Völkerrechıssubjekten, de-

4) Texce in: Günter Neuberger/Michael Opperskalski: ClA in Westeurop2, Bornheim 1981. S. 93 ff. Informacionen hierzu finden sich zuch in: Bläetle (Sturtgar) 3/1982. Forokopic im Besitz des Verfassers.

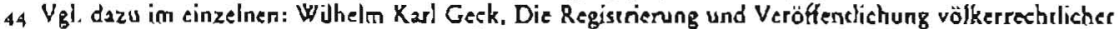
Verträge, in: Zeieschrift für zusłändisches Öffentliches Recht und Völkectecht, Band 22 (1962), 5. 1 is ff: $\mathrm{Karl}$ Zemanck, Geheimverträge. in: Strupp/Schlochauet, Wörerbuch des Völkerrechus, Band I, 1. Aullage $1962,5.633$ \%.

4s O. Gopper, Der Völketound, 1938, S. 47 ff: ein Beispiel dalür ise der deuesch-sowjecische Nichungrifls. pakt vorn 23. 8. 1939 mil dem geheimen Zusazabkommen über die • Behandlunge Polens.

46 Vgl. Kael Zemanek, a, 3. O., S.634. 
nen die Fähigkeit zum Abschluß völkerrechrlicher Abmachungen fehlt; Abkommen zwischen vertragsfähigen Völkerrechtssubjekten werden dann nichr erfaßr, wenn sie nicht dem Völkerreche unterstehen, sondern ecra auf ciner privatrechelichen Grundlage beruhen.

Auch einseicige Erklärungen völkerrechdichen Charakters sind registrierungspflichiig, wenn sie im Ergebnis eine vertragliche oder vertragsähnliche Bindung bewirken."

Sollen somir Vereinbarungen zwischen der Bundesregierung und der US-Regierung oder Naco-Srellen exiscieren, die auf eine völkerrechtliche Bindung der Bundesrepublik im Hinblick auf die Scationierung spezieller Waffensysteme in der Bundesrepublik abzielen, so unterliegen auch diese der Verpflichtung zur Registrierung nach Arrikel 102 der Charta der Vereinten Nationen. Da sowohl die Bundesrepublik als auch die USA Mirglied der Vereineen Nacionen sind, könneen, da solche Geheimabkornmen nach bisherigem Erkennenisstand nicht bei den Vereinten Nationeo registriert sind, weder die USA noch die Bundesrepublik aus diesen Vereinbarungen vor irgendeinem Organ der Vereinten Nacionen Rechtsfolgen geltend machen; zu den Organen der Vereinten Nationen gehört auch der Ioternationale Gerichtshof.4" Vor allen anderen Srellen, die nicht Organe der Vereinten Nationen sind, sollen sich nach verbreiteter völkerrechtlicher Auffassung jedoch die Vertragsstaaren in recht. lich wirksamer Weise auch auf nicht-registrierte Abkommen berufen können."9 Die Bindung der Vertragspartner an solcbe Geheimabkommen (jenseirs der Organe der Vereinten Nationen einschließlich des Internationalen Gerichtshofes) ist daher zuvörderst eine Frage des politischen Willens der Parner des (Geheim-) Abkommens. Ob der (geheim-) abkommensbrüchige Sraat darüber hinaus ein »völkerrechtliches Deliks « begeht, ist in der völkerrechtlichen Literarur ungeklärt. Angesichts der weitgefaßren Registrienungs- und damic Publizierungspflicht in Arrikel 102 der Charta der Vereinten Nationen dürfte die Aowendung der völkerrecbrlichen Grundsätze der *völkerrechtichen Verantwonlichkeic « auch auf nicht registrierte Geheimverträge jedoch abzulehnen sein: Wenn auch die Zulässigkeic einseiriger Gegenmaßnahmen des „Verlerzten * durch das moderne Völkerrecht bereits erheblich eingeschränkc ist, bliebe sonst doch dem $>$ Verletzten« als weitestgebende Form der Selbschilfe das Rechr zur sogenannzen Repressalie, d. h. die Vergelrung der Nichreinhaituog eines nichtregistrierten Abkommens mit einer weiceren Völkerrechtsverlerzung. ${ }^{10}$

Auch die Tarsache, daß die Einführung der Regismierungs- und Publizitätspflich für völkerrechtliche Veräge und Abkommen aller Art letzulich aus der historisch begründeten Kricik an der "Geheimdiplomatie* des 19. Jahrhunderts und der Jahre unmitrelbar vor dem 1. Weltkrieg resultiert," macht eine (indirekte) Anerkennung von nicht-registrierten Geheimabkommen durch die Völkerrechesgemeinschaft mehr ais problemarisch.

Die Frage, in wieweit in der Bundesrepublik das Pariament am innerscaclichen Verrragsabschiußverfahren zu bereiligen ist, kann hinsichdich dieser (möglichen) Geheimabkommen nichı abschließend geklärt werden, da dazu der Inhait dieser Ver-

47 Vgl. dazu u. 2. Withelm Karl Geck, Die Registrierung und Veröffentlichung völkerrechdicher Verträge, 2. 2. O., S. 142 If, 1486 .

$48 \mathrm{Vgl}$ dazu u. 2. Paul Guggenheim, Lebrbuch des Völkerreches, Band i, Basel 1948, S. 81 f.

9 Vgl. u. a. Geck, Registrierung von Vereragen, in: Strupp/Sehlochauer. Wörecbuch des Völkerreches. Band III, 2. 2. O.. S. 96 If. 98 .

so Vgl. allgemein zur Repressalie Knut losen, in: Menzel/lpsen. Völkerreche, 2. Auflage 1979, S. 347.

s1 Vgl. dazu u.a Ernst-Günter Gnudeke, Geheimdiplomasie, in: Surupp/Schlochauer, Wöruerbuch des Volkerrechis, 2. Aufage 1962, S. 632 f: Herbert Kroger u. a. (Autorenkollekciv), Volkerreche, Köln 1973, S. 2361. 
träge bekannt sein müßre. Denn nach Arrikel sg Abs. 2 des Grundgesetzes bedürfen lediglich solche Verträge, nrvelche die politischen Beziehungen des Bundes regeln oder sich auf Gegenstände der Bundesgeseczgebung beziehen $\propto$, der $*$ Zustimmung oder der Mitwirkung der jeweils für die Bundesgeseızgebung zuständigen Korperschafren in der Form eines Bundesgeserzesa. Nach der Rechesprechung des Bundesverfassungsgerichrs wird ein Verrag noch niche dadurch zu einem "politischen a im Sinne des Arrikels 59 Abs. 2 Sacz I Grundgesetz, daß er sich ganz allgcmein "mit öffendichen Angelegenheiten, dem Gemeinwohl oder den Staatsgeschäfren* befaßt. ${ }^{\text {'2 }}$ Hinzukommen muß nach Ansicht des Bundesverfassungsgerichrs vielmehr. •daß die Existenz des Staates, seine territoriale Integrität, seine Unabhängigkeit, seine Stellung oder sein maßgebliches Gewicht in der Staarengemeinschaft durch den Verragg selbst berühtrt werden «.3" Politische Verträge in diesem Sinne seien vor allem solche, die darauf gerichter sind, wdie Machtstellung der Bundesrepublik anderen Scaaten gegenüber zu behaupren, zu befestigen oder zu erweiterna." Bündnisse, Neutralitärs- und Nichtangriffspakte sollen nach allgemeiner Meinung hierunter fallen. Sie unterliegen daher als "politische Verträge dem $Z$ wang parlamentarischer Zustimmung (Arr. s9 Abs. 2 Satz x GG) und damit dem Verbot, daß sie als Geheimverträge abgeschlossen werden. ${ }^{44}$ Allerdings werden sogenannte ngeheime Verwalcungsabkommen ₹ von diesem Parlamentsvorbehalt nicht berühr. " Die Abgrenzung zwischen *Abkommen $\propto$ und "politischen Verträgen $\propto$ im Sinne des Art. 59 Grundgesetz ist sehr unklar. ${ }^{36}$

Letzdich kann eine rechtliche Zuordnung der jeweiligen völkerrechtlichen Vereinbarung zu der Karcgorie "policische Verträge " oder "Verwaitungsabkommen* lediglich vom Inhalt derselben her vorgenommen werden, bei »Geheimabkommen ein fast aussichrsloses Unterfangen,

Bei Geheimabkommen freilich, dic die Stationierung und ggf. den Einsacz von Massenvernichtungswaffen unter bescimmten Vorausserzungen im Bundesgebier gestatten, ist jedoch praktisch nicht vorstelibar, wie solche Vereinbarungen ndie Existenz des Staates, seine territoriale Integrität, seine Stellung ( $B V$ erfG) nicht *berühren * solltren. Zurückhaltend ausgedrückt: Es dürfre nicht ganz einfach sein, verfassungsrechrlich die Umgehung des parlamentarischen Zustimmungsvorbehaltes vor Art. 99 Abs. 2 S. I Grundgesetz bei Geheimabkommen dieser Art zu legirimieren.

\section{Verfassungsrechtliche Grenzen: Stationierung nur mit Zustimmung des Gesetzgebers?}

Wolfgang Däubler hat in seiner kürzlich veröffenelichren Scudie "Scarionierung und Grundgesetz die Auffassung verreten, der Gesetzgeber müsse selbst die Kriterien aufstellen, "nach denen die Regierung über die Stationierung neuer Waffensysteme entscheiden könnte «; dies gelte jedenfalls für solche Angelegenheiten, die eine vergleichbare Bedeurung wie der Bau eines Kernkrafowerks besäßcn. Das wgesczzliche

\$12 BVerfGE 1, 380.

\2 BV CRGE 1, 380.

is BVerfGE I, 380 f.

s4 V $V_{\mathrm{g}}$. dazu ecwa Ondolf Rojahn, in: Ingo von Münch (Herausgeber), Grundgeselzkommentar, Rd. Zilf. 23 i zu Ar. 59 .

ss Vgl. dazv etwa Geck, in: Feseschrift für Gebharde Müller, 1970, S. 96, 102; Rojahn, 2. 2. O.. Rd. Ziff. 1s zu Are. 39.

s6 Vgl. dazu u. a. Bülck, Verwaltungsabkammen, in: Sinupp/Schlochauer, Wönerbuch des Völkerteches. Bd. 3. 1962. S. 562 ff; Hienseorfer, Die 2uswarige Gewals des Deueschen Bundestages. Dissertacion Marbung 1968 . 
Programm* müsse so detailliert sein, *daß nicht das Schwergewicht der Entschei-

dung bei der Exekurive* liege; Pauschalermächtigungen skönnen nicht genügen $\propto$; der Gesezzgeber sei gezwungen, wSicherheirsvorkehnungen gegen Unfälle vorzusehen und andere wesentliche Fragen zu regeln ${ }^{17}$

Auch die frühere Bundesregierung hat durch den Parlamentarischen Staatssekretär im Bundesjustizministerium Hans de With auf die Frage des SPD-Abgeordneten Horst Sielaff geäußer: ^Ob es im Lichce der neucren Rechtssprechung des Bundesverlassungsgerichis zum Vorbehalt des Geserzes über die Zuscimmungsgeserze zu den genannten Abkommen hinaus (Deutschlandvertrag und Aufenthaltsvertrag) für die Lagerung (chemischer Waffen) einer besonderen geserzlichen Emächrigung bedarf, wird im Hinblick auf die der Bundesregierung in jüngster Zeit übersandre gutacheliche Scellungnahme (des DGB-Landesbezirks Rheinland-P(alz) derzeit von den bereiligren Ressores gepnifta." Sicher ist, dals das Grundgeserz keine allgemeine Besummung enchäle, derzufolge der Geserzgeber über die im Normtext enthaltenen Vorbehalte hinaus besrimmte Fragen seibst entscheiden muß und sie nicht der Exckurive überlassen darí. Dennoch har das Bundesverfassungsgericht seir Beginn der siebziger Jahre vor allem im Schulrecht den Versuch unternommen, die tradicionelle Auffassung vom Gesetzesvorbehalt "dem heutigen Verfassungsverständnis» anzupassen." Nach überkommenem *klassischem « Verständnis bedeurete der *Gesetzesvorbehalt火, daß die staalichen Behörden dort einer geserzlichen Errmächtigung bedürfen, wo sie in die Rechre der Bürger ( $*$ Freiheit und Eigentum a) eingreifen. Nach Auffassung des Bundesverfassungsgerichts, die von zahlreichen Stellungnahmen in der juristischen Fachliteratur vorbereitet worden war, ${ }^{60}$ mußte diese alze Doktrin *auf ein neues Fundamen gestell $\ll$ werden. Die vom Bundesverfassungsgeriche aufgegriffene neue Dokerin postuliere, $*$ daß die Entscheidung aller grandsäzzlichen Fragen, die den Bürger unmitcelbar betreffen, durch Geserz erfolgen muß, und zwar losgelöst von dem in der Praxis fließender Abgrenzungsmerkmal des Eingriffs Dem vom Parlament beschlossenen Geserz komme ngegenüber dem bloßen Verwaltungshandeln die unmitrelbarere demokrarische Legitimacion zu, und das partamentarische Verfahren (gewährleisze) ein höheres Maß an Öffentlichkeir der Auseinandersetzung und Entscheidungssuche und damit auch größere Möglichkeiren eines Ausgleichs widerscreirender Intcressen $\alpha_{,}{ }^{61}$

Dieser neue Ansatz wurde rasch mit dem Begriff "Wesentlichkeitstheorie $\alpha$ eriketriert. Er ist zentriert um den gemeinplätzlichen Topos, die $\times$ Binsenweisheit . .., daß ... die wirklich wichtigen Dinge in einem parlamentarisch-demokratischen Staatswesen vor das Parlament gehör(t)en . $^{6 x}$

Läßt sich gegen diese Auffassung dann kaum erwas einwenden, wenn sie als politische Option aufrit, so besteht die Problematik der "Wesentlichkeitstheorie* vor allem darin, daB in ihr den Gerichcen die Befugnis zugewiesen wird, darüber zu entscheiden, was nwirklich wichtig* und swesentliche (= regelungsbedürfrig) ist. Hatte die tradierte, an der Perspekrive des der scaadichen Gewalt unterworfenen Individuums orientierce Konzeprion des . Geserzesvorbehaltse ibre relarive Festig-

S7 Däubler, Stacionicrung und Grundgeseiz, S. 227 ff.

is Bundestagspronakull g/107, ziliere nach: Rechespolitischer Dienst (RPD). 64. Lieferung vom 23. 7. 1982 .

is V Vl. ela BVeriGE +0, $237(2+9)$.

6o Vel. dazu ęwa Thomas Oppermann. Nach welehen rechelichen Grundsärzen sind das öflentliche Schulwesen und die Stellung der an ihm Beteiligien zu ordnen? Gutachten $C$ zum s. Deutschen Juristenug, Nünchen 1476, C 44 fi; krivische Anmerkungen dazu etwa bei Diecer Deiseroth, Informationsberiche

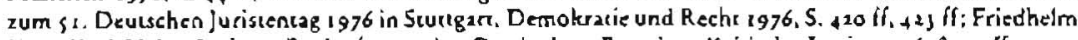
HaseiKarl-Heinz Ladeur. Rechesicagen dec Curriculum-Revision. Kriviscbe Jusciz 1976, S. ig If:

61 BVeriGE $40,217(249)$

62 BVertGE $4 \%,+6(79)$, 
keit daraus gewonnen, daß sie sich darauf beschränkte, die rechtlichen Voraussctzungen staatlicher Eingriffe in individuelle Rechrspositionen zu umrcißen, so entbehre die neue, allgemein auf das " Wesentlichea und * wirklich Wichrige- abhebende Dokurin eines vergieichbaren Halts. ${ }^{6}$

Die Schwierigkeit, das "Wesentlichen zu bestimmen, hat denn auch in der Litcratur Fragen und Bedenken provoziert. ${ }^{\text {s4 }}$

Die Rechtsprechung selbse liefert über die allgemeinen Begriffe , wesencliche und "wichuig" hinaus lediglich Allgemeinheiten: "Ob eine Maßnahme wesentlich ist und damir dem Parlament selbst vorbehalten bleiben muß ..., richtet sich zunächst allgemein nach dem Cirunigeserz $\propto{ }^{64}$

Frcilich sagr das Grundgeserz, wie beseits oben angedeuter, zu dieser Frage lediglich in einigen Fällen konkret, nirgendwo aber allgernein etwas aus. Weirer heißı es in der Rechrsprechung des Bundesverfassungsgerichts, sder Schutz der Grundrechee a ver-

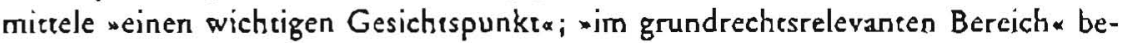
deute wesentlich in der Regel wwesentlich für die Verwirklichung der Grundrechre $\alpha^{66}$

Die mir Tautologien und Unklarheiten gespickten Wendungen belassen somit also den Gerichten einen außerordentlich weiten Bewercungs- und Definitionsspielraum, der noch dadurch zusärzlich vergrößert wird, daß bereirs auf dicser allgemeinen Ebene in ebenso unbestimmter Weise Reserven formuliert und entgegengeserzte, die Reichweite des Geserzesvorbehaltes wieder limitierende rherorische Gesichtspunkte inszalliert werden: "Bei der Abgrenzung (zwischen wesentlichen und unwesentlichen Fragen) wird man mit großer Behursamkeit vorgehen und sich die Gefahren einer zuweirgehenden Vergeserzlichung ... vor Augen halten müssen * (ebd.). Das Bundesverfassungsgericht selbst bat in der sogenannten Kalkarentscheidung zum "Schnellen Brüter a deutlich gemachr, dem Parlament komme kein wallumfassender Vorrang * bei grundlegenden Entscheidungen zu; das Grundgeserz habe vielmehr bei weicreichenden politischen Entscheidungen wie etwa der Aufösung des Bundestages nach Artikel 68 oder der Aufnahme und dem Abbruch diplomarischer Beziehungen zu anderen Staaecn die Komperenz anderen Verfassungsorganen vorbehalten ${ }^{67}$ es gebe keinen "Gewaltenmonismus * in Form eines gencrellen Parlamentsvorbehaltes.

Im Falle der Seacionierung neuer amerikanischer Waffensysteme in der Bundesrepublik, die leczdich auf einer natointernen Willens-und Entscheidungsfindung beruht, ließe sich leicht cine gesetzgeberische Entscheidung finden: Die Zuscimmungsgesetze zum Generalvertrag und zum Aufcnthaltsvertrag sowie der vom Gesetzgeber ratifizierte Beitritt zum Nato-Vertrag.

In den Kategorien der "Wesentichkeitsrechtssprechung * liefe deshalb alles darauf hinaus, wie bestimmt eine soiche gesetzliche Regelung sein muß, wic weit sie ins Einzelne zu gehen und die Praxis der administrativen Apparace im konkreten Fall zu determinieren har. Wer sich daran machr, aus der Rechrsprechung des Bundesverfassungsgerichts oder aus der einschlägigen Literatur generalisierbare Anrworten auf diese Frage zu exhakten, wird Schifforuch erleiden. Die in den allgerneinen Bestimmungen der * Wesentlichkeirstheoriex enchalrenen Unklarheiten werden dadurch noch porenziert, daß auch dic Kriterien, an denen die Bestimmrheit des regelnden

6) Vgl. zum Folgenden Dieter Deiseroth/Friedhelm Hase, Der =Schnelle Brüter- vor Gericht. Demokratic und Recht 1979. S. I Is If. 114 il.

64 Vgi. z. B. Kisker. Njog 1977. S. 1 yly ff.

6s BVerfGE $47 . \$ 6(49)$.

$66 \mathrm{BV}_{\mathrm{CH}}\left(\mathrm{GE} \mathrm{4}_{47,46}(79)\right.$.

6) VEl. BVerfGE 49 , 99 (12s). 
Geserzes gemessen werden soll, nur in unbesummeer, gegensützliche Aspekıe zusammenschließender und entgegengeserzte Entscheidungen porenciell legicimierender Weise umschrieben werden. So soll nach der höchstrichterlichen Rechtsprechung erwa die Einführung des Sexualkundeunterrichts oder die Umscellung der schulischen Leistungsbewertung auf das Punktesystem *wesentlich a und *wirklich wichcig«, danit einer parlamencarischen Regelung bedürfrig sein, die smöglichen Folgewirkungen, Gefahren, und irreversiblen $Z$ wänge, die sich an den Bau sSchneller Brürer knüpfen könnten a, jedoch nicht. ${ }^{\text {bi }}$ Einerseirs soll nach diescr Rechrsprechung der Geserzgeber sselbst entscheiden, ob Sexualkunde fächerübergreifend oder als besonderes Unterrichrsfach dargeboten * wird; ${ }^{* 4}$ andererseics darf aber der Exekutive die Bescimmung des "Restrisikosa überlassen werden, das bei der Errichrung von Kernkraftwerken wie dem schnellen Brürer « hingenommen werden muß.”

Diese - mit Verlaub - in leeren Begriffshülsen aufgebauten Gegensäıze zwischen *grundlegenden Entscheidungen « und bloßen *Einzelheiten a ermöglichen keine juristische Abgrenzung, die zukünftige Entscheidungen der Gerichte auch nur ansarzweise vorherseh- und berechenbar machen könnte. Die Begriffe können zwanglos variiert, enger oder auch weiter gefaßst und umstandslos gegeneinander verschoben werden: Die * Wesentlichkeitstheorie weist damir die eigencliche Enrscheidung dem an den Fronten des einzelnen Falles oder des Konflikss orienciercen Wahrnehmungshorizont der Gerichte zu, in deren Problemperzeption und bewernug erst wWesentliches* vom «Unwesentlichen «, *Grundlegendes* vom »Einzelnen* separiert werden kann. Ganz offenbar hängr es von der Eigenart der jeweiligen Konfliktfelder und "Sachbereiche* $a b$, ob und wie weit sie in der gerichtlichen Bewertung als - wesentlich « und * regelungsbedürfrig « erscheinen." Die Frage danach, ob nach die. ser w Wesenzlichkeits *-Rechtsprechung ein bestimmter Problembereich bereits weine parlamentarische Leitentscheidung mit hinreichender Bestimmtheic erfahren har, ${ }^{2}$ kann deshalb niche durch eine philologische Ausdeutung einzelner Urteile und Urreilspassagen ausreichend beantworter werden. Vielmehr müssen die jeweils agierenden staaclichen Apparate und deren spezifisch juristische, politische und ideologisehe Handlungsbedingungen einbezogen werden. Denn die klarseen und präzisesten Rechrsnormen können ins Schwimmen geracen, können als widersprüchlich, lückenhaft oder unzureichend erscheinen, wenn die in ihnen vorausgeserzten und angesprochenen Behörden nicht im Stande sind, die Rechtsbegriffe in einer konsisrenten, für die Enrscheidungsabnehmer akzeprablen Praxis einheitlich anzuwenden und in einem bescimmren Sinne festzulegen. Umgekehrt können die allgemeinsten und unklarsten gesetzlichen Regelungen - ein Beispiel ist die polizeiliche Generalklausel ("Öffentliche Sicherheit und Ordnung*) - als hinreichend bessimmt angesehen werden, wenn sie an Behörden adressierr sind, die auf einer scabilen ideologischen und politischen Grundlage eine weindeutige* Praxis enrfajten können. Gerichre sind was gerade auch die Genese der Wesentlichkeirsrechrsprechung eindrucksvoll demonstriert - weniger auf das widerspruchsfreie Durchhalten von dogmatischen Konstruktionen, als vor allem auf Jas Finden von Abnahmebereitschaft für ihre Dezisionen bedacht. Nur so können sie offensichtlich die Funktion erfüllen, - Rechrsfrieden « wiederherzustellen. Dies ist zwar rechrlich nirgendwo ausdrücklich

68 So das 8 VerfGE in seiner Ensscheidung zum =Schnellen Brïue a in: EuGRZ 1979, S. 583 ff. (s63); anderer Aulfassung OVG Munstcr, Ureil vom 18.8. 1977, S. 18 If im Original.

69 V 1 l. dazu BVcrGGE $+7.46(8)$ ).

70 BVeriGE in: EUGRZ 1978, S. 353 It. 566.

7 So duch ausdnicklich des BVeqfGE in der Kalkar-Enischeidung vom 8. 8. 78, EuGRZ 1978, S. 553 ff, s6\}.

72 BVerfGE $47,+6(8)$ ). 
normiert; es ist aber, wic sozialwissenschafeliche Untersuchungen nahelegen," "Geschäfesgrundlagen gerichtlicher Verfahren.

Gerade weil fast alle politischen Kräfre die Verankerung der Bundesrepublik in dem Naro-Bündnis weithin vorbehalclos unterstüczen und insofern eine relativ stabile ideologische und politische Grundlage einer neindeucigen Praxis existierr, ist nur schwer vorstellbar, daß etwa das Bundesverfassungsgericht mit Hilfe der * Wesentlichkeicstheorie* dieser exekurivischen Praxis die verfassungsrechtliche Grundlage bestreiten würde. Selbst wenn es auf diesem Gebiet rechtliche Fragezeichen serzen würde, dürfte die Prognose nichr fehl gehen, daß das Gericht, nicht zuletzr aus Gründen der notwendigen Akzeptanzfindung, dem Gesetzgeber das "Nachbessern* leicht machen würde.

Nichc übersehen werden darf dabei, daß die Befassung des Bundesverfassungsgerichts mit dieser Frage und eine entsprechende »Absegnung* der Grundlinien der exekucivischen Praxis jener einen deutlichen Zugewinn an Legitimation verschaffen dürten: Das Gütesiegel sverfassungsrechtlich zulässig w würde dann nichr zum ersten Mal politische Eigendynamik entwickeln. Diese politische Kosten einer Anrufung des Bundesverfassungsgeriches in dieser Frage müssen deshalb wohl kalkulier wcrden.

\section{Wer entscheidet über den Einsatz der in der Bundesrepublik stationierten (nesen) atomaren Waffensysteme?}

Die Bundesregierung hat in der Broschüre des Presse- und Informationsamtes nAspekte der Friedenspolicik im Juni $19 \$_{1}$ im Tone größcer Selbstverständlichkeic darauf hingewiesen, daß mauch die neven Mittelstreckenwaffen (also Pershing II und Marschflugkörper) . . . nur von Amcrika produzierr (werden) und . . . in amerikanischer Verfügungsgewalt bleiben. Uber ihren Einsazz kann ausschließlich der amerikanische Präsident entscheiden, (Die Bundesrepublik har) ... keinen, Finger am Abzugie."

Es bedarf keiner näheren Darlegung, daß die Entscheidung über den Einsarz von Nuklearwaffen in der Bundestepbulik oder vom Boden der Bundesrepublik aus einer Entscheidung über die existenzielle Vernichtung der bundesrepublikanischen Bevölkerung gleichkommr. Denn spärestens seit der sogenannten von WeizsäckerStudie ("Kriegsfolgen und Kriegsverhürung*) aus dem Jahre $1970 / 72$ ist die mit einem großen wissenschaftlichen A ufwand abgesicherte Erkenntnis bekannc, daß die Bundesrepbulik im Kriegsfalle, wenn also die *Abschreckungskomponente" der geltenden Nacoscrategie der " llexible responsen versagt hat, mit militärischen Mitceln nicht zu verteidigen ist. Die Ergebnisse dieser Studie sind bisher weder von offizieller Seite der Nato noch vom Verteidigungsministerium oder in der wissenschaftlichen Diskussion in Frage gestellt worden. Sie lauren im Detail (zusammenfassend): - Unter dem Aspekt der Kriegsfolgen ist zu sagen, daß ein konventioneller Krieg, wenn er unser Land in wenigen Tagen schnell durchzieht, nur begrenzeen Schaden bringen würde, während der vielleicht denkbare Fall eines in unserem Lande hin-

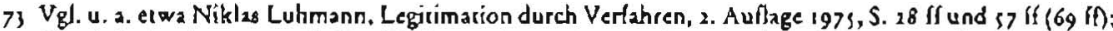
Kiescheic, Justizapparate als Konflikrlösungsinstanz?. Demokrarie und Reche 1979. 5. I fl, if: vgl. dazu auch Theodor Maunz, Deutsches St2atsrecht, 20. Auflage :975. $\$ 10$ II 3 (S. 280); Stürner. Schuex des Gerichtsverfahrens vor öffentliche Ein/fußnnahme?. Juristenzeirung 1978. S. 16 ! If (166): Aus dec Ríchterperspekúve z. B. Heusinger, Rechsfindung und Rechisforibildung im Spiegel richterlicher Erf́ahrong. 1975, S. $\$ 9$.

74 Ebd., S. $1 \%$ 
und hergehenden oder Gesigefahrenen, lang andauernden konventionellen Krieges

uns Zerstörungen bringen würde, die an den Beispielen der Sowjetunion im zweiten Weitkrieg, Korea und Vietnam nur unvollkommen abzulesen sind und die, sowohl wegen der größeren Ausmaße wie wegen der größeren Verleczlichkeic eines Industrielandes für unsere wirtschaf́liche Fortexistenz lebensgefährlich werden könnten *". - Bercits der Einsatz eines Bruchteils (je nach Kriegsbild erwa 2 bis $10 \%$ ) der in Europa vorhandenen Acomwaffen bedeutec einen vernichcenden Schlag für die Bundesrepublik. Diese Aussage ist unabhängig von den getroffenen Schutzmaßnzhmen. Sie gilt auch noch bei einer Beschränkung auf einen Bruchteil allein der takiischen Atomwaffen - ebenso gilt sie bei dern ausschließlichen Einsazz eines Bruchteils strategischer Waffen “. ${ }^{76}$ Angesiches dieser sicherheirspolitischen Situation war es naheliegend, daß die Bundesrepublik ebenso wie die anderen Nato-Sraten seir langem daran interessiert war, Einflub auf die nukleare Strategie zu gewinnen. Lange Zeit waren die Europäer jedoch mehr an einer Gewähr dafür inceressiert, daß die USA Kernwaffen einsetzen würden, wenn es im Inzeresse der europäischen Bündnismitglieder notwendig erschiene, als an der Verhürung eines vorsehnellen amerikanischen Kernwaffeneinsatzes," Aus diesem komplizierten und in sich widersprüchlichen politischen Prozeß entwickelte sich eine inscitutionalisierte form der natointernen Konsultation, die freilich explizit die Entscheidungsbefugnisse des US-Präsidenten über den Einsarz von amerikanischen und des bririschen Premierministers über den Einsatz von brivischen Nuklearwaffen im Konflikt\{alle unberühr ließ.

\section{Das Nato-Konsultationsverfabren}

Von Bedeurung für das Naco-Konsultarionsverfahren sind vor allem die Beschlüsse der Naro-Rats-Tagung yom Mai 1962 in Athen und die auf eider belgisch-amerikanischen Studie beruhenden $*$ Richrlinien für das nukleare Konsultationsverfahren «, die auf der Naro-Rars-Tagung im Dezember 1969 vom Defense Planing Committee verabschiedet worden waren.

In Athen war im Jahre 1962 beschlossen worden, einen aus den ständigen Delegierten der fünfzehn Nato-Staaten bestehenden *Nuklear-Ausschuß einzurichten, wum allen Mirgliedsstaaten eine volle Bereiligung an der Konsultation über die nukleare Verteidigungspolitik zu ermöglichen $\alpha^{7^{8}}$

Dem Nuklear-Ausschuß sollten die USA Informacionen über Größe und Lage der Ziele sowie über die Zerstörungskrafr der Waffen geben, und ihn solkren sie wegen der Anwendung der Waffen konsultieren. ${ }^{\$}$

Die 1969 beschlossenen $*$ Richdioien für das nuklease Konsulcationsverfahren a sind screng geheim. ${ }^{80}$

Nach vorliegenden Informationen sind die Naco-Staacen jedoch dahin übereingekommen, daB die USA verphlichtet sind, vor der Frcigabe und dem Einsatz von für die Nato vorgesehenen rakrischen Nuklearwaffen (theater nuclear forces) bis zu

\footnotetext{
7s Vgl. C. F. von Weizszcker. Einleirung zu -Kriegslolgen und Krieysverhútung=, 2. Auflage 1971, S. 9. $76 \mathrm{~V}$ l, Ph. Sonniag, Machemalisthe Analyse der Wirkungen von Kernwallenexplosionen in der BRD, in: von Weizsieker, 2. 1. O., S. 198: vgl. zu den Diskussionen über die mulise-politisehe Ineeressenlage der Bundesrepublik, Dieter Mahneke. Nukleare Mirwirkung, Berlin 1971, S. 14 lf. 
einem gewissen Grad ein Konsultationsverfahren durchzuführen. „Das Zustandekommen eines solchen Konsultations-Procedere serzt voraus, daß der US-Präsident niche durch Zeic und Umstände (time and circumscances permitring) zu raschem Handeln gezwongen wird $\alpha . "$

Die Pflicht zur Konsulcation der Nato-Verbündeten durch den US-Präsidenten, also $\operatorname{der} Z$ wang, diese anzubören, steht mithin uncer dem ausdrücklichen Vorbehalt, daß *Zeic und Umseände es erlaubenk. Erforderz jedoch beispielsweise das mationale Inceresse der USA nach Auffassung des US-Präsidenten ein »sofortiges Handelna. »so dürfte die amerikanische Entscheidung ohne Beceiligung der europäischen Verbündeten getroffen werden. ... Die Vereinigten Staten und auch jeder andere Nato-Partner, der über eigene Nuklcarwaffen verfügt, rvie Großbritannien und Frankreich, sind frei, dann ohne vorherige Konsultazion der Verbündeten eine unabhängige Entscheidung zu treffen, wenn die Lage (z. B. im Falle eines Überraschungsangriffes) kein enesprechendes Konsultationsverfahren zuläßt bzw. eine sofortige Reaktion (auch in $*$ subjektiver Perzeptiona) nörig isc. ${ }^{8 x}$

Im Regelfall soll das Konsultationsverfahren jedoch in der Weise prakciziert werden, daß jedes Ersuchen einer Regierung oder eines hohen Naro-Befehlshabers um die Freigabe von Nuklearwaffen und jede Möglichkeir des Einsatzes durch eine $\mathrm{Nu}$ klear-Macht sofort den einzelnen Nato-Regierungen und dem DPC (Defense Planing Committee) mitgeteilt werden. Die Scellungnahmen dieser Organe werden der betroffenen Nuklear-Macht übermitrelt, und die Entscheidung dieser Machs wiederum soll den verbündeten Regierungen, dem Nato-Rat und den hohen NatoBefehlshabern zur Kennunis gebracht werden.

-Die Freigabe der Nuklear-Waffen zum Einsatz wiederum kann allerdings nur durch den US-Präsidencen bzw. den britischen Premierminister eriolgen . Geben diese die Nuklear-Waffen frei, so stellt *die Freigabe gleichwohl . . keinen Einsatzbefehl dar. Der SACEUR (Oberster Alliierter Befehlshaber Europa) behält damit die Möglichkeir, die Nuklear-Waffen nach seinem Ermessen einzuserzen. $k^{8_{3}}$

Da jedoch SACEUR sowohl Oberster Nato-Befehlshaber als auch Oberkommandierender der US-Streickräfte in Europa isc, ist er in dicser seiner zweiten Eigenschaft den Weisungen des US-Präsidenten auf dem nnationalen Befehlsstrang < unmizeibar unterstellc. Insofern obliegr auch nach der Freigabe der Nuklear-Waffen zum Einsatz der konkrete Einsazabefehl weiterhin dem US-Präsidenten. Unklar ist freilich, in welcher Weise sich die Unterstellung des SACEUR unter die NATO-Dienststellen im Konfliktfalle auswirken wird, wenn also z. B. die entsprechenden NATOOrgane (mchrheitlich) gegen einen Einsatz nuklearer Waffen votieren sollten.

Im Ergebnis bedeutet dies, daß die Entscheidung über den Einsarz der neuen amerikanischen Miccelstreckenwaffen, falls sie erst einmal in der Bundesrepublik stationier sind, nur sehr bedinge vom NATO-internen Konsultationsverfahren abhängig ist; die Encscheidung über den Einsacz dieser Waffen und damic über Leben und Tod von Millionen Menschen in Europa fällt also nicht in Europa, sondern in den USA.

81 $V_{\mathbb{R}}$, Dierer Wolf. Die Encseheidung úber den Einsazz von Nukleanarafen, in: Aus Politik und Zeitgeschichse. B 18/79. S. 2911.37 .

82 Vgl. Wolf. 2. 2. O., S. 18.

8) Vgl. Wolf, 2. 2. O., 5. 39. 
Nach der amerikanischen Verfassung hac der Kongreß zwar die Befugnis, namens der USA anderen Staaten den Krieg zu erklären. Der Präsident ist jedoch »Oberbefehlshaber des Heeres und der Marine sowie der Miliz der einzelnen Staaren (Ar. II, Sec. 2 der US-Verfassung). Danach kann der Präsident alle takrischen und strategischen Anordnungen und Maßnahmen treffen, die den Einsatz von Menschen und Marerialien betreffen. Auch vor der formellen Kriegserklärung durch den Kongreß gibt die amerikanische Verfassung dem Präsidenten die ausdrückliche Befugnis, Überraschungsangriffe auf die USA abzuwehren ( zählt insbesondere auch die Encscheidung darüber, ob ein Ưberraschungsangriff auf die USA vorliegt und welche Maßnahmen ergriffen werden müssen. In der verfassungsrechrlichen Liceratur der USA wird diese Befugnisnom heuce auch dahin ausgelegt, daß ein solcher wÜberraschungsangriff auf die USA auch dann vorliegen soll, wenn amerikanische Staatsangehörige und ihr Vermögen im Ausland angegriffen werden." Umseritcen ist freilich, ob diese wsudden repulse power auch dann zum Zuge kommt, wenn es darum geh, wÜberraschungsangriffe auf (NATO-) verbündete Stazten abzuwehren. Da hier im rechrlichen Diskurs keine eindeutige Klärung bisher erzielt wurde, dürfte schon aus diesem Grunde die präsidentielle Befugnis keine rechtlichen Restriktionen zu gewärtigen haben. Im Klartext: Der US-Präsident (mit seinem Beraterstab) entscheider praktisch darüber, ob ein Überraschungsangriff auf die Verbündeten vorliegt und ob dies seiner Ansicht nach einem Überraschungsangriff auf die USA gleichgesteilt werden kann.

Die Entscheidung über Leber und Tod von Milionen Menschen in Europa liegt damit (auch) nach amerikanischem Verfassungsrechc in den Häoden des US-Präsidenten, der diese - was sich näheren Überprüfungen entziehc - an bescimmte Befehlshaber zumindest paruiell delegieren kann."

84 Vgl.dizu ecwa Don Wallace, The War-Making Powers: A Constitucional Flaw?, in: Cornel Law Revicw Band $37(1972)$, S. 719 ff, S. 337 . Allgemein zu den Kriegsrecheen des US-Präsidenten: Dieter Wolf, Um dic Kriegsvollmache des amerik anischen Präsidenten. Der W/apowers Ace vom 7. Nov, 1971, in: Europ2Archiv 1974, S. 216 ff: Dieler Woll/Manfred A. Dauses, Die Kriegsreche in den Vereinigen Staaten. Anzlyse der vertassungsrechelichen und politischen Auseinandersetzung zwischen Prasidene und Kongres um die - Warpowerse, Berlin 1979, S. $\$ 2$ if. 68 If.

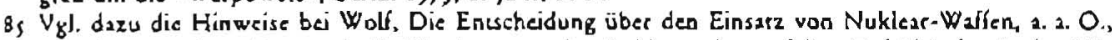
S. l1 $_{1}$. Die weirere Frage, ob die Einriumung oder Duldung einer solchen Befehlsbefugnis des US. Peasidenen mil Wirkung fur das Hoheisgebiet der Bundesrepublik durch die Bundesregierung nach dem Grundgesecz zulissig ist (- Ubereragung von Hoheitsrechen. 7), rouß einer speziellen Untersuchung vorbehalien bleiben. 